\title{
Probing the IGMF with the Next Generation of Cherenkov Telescopes
}

\author{
M. Fernández Alonso, A. D. Supanitsky, and A. C. Rovero \\ Instituto de Astronomía y Física del Espacio (IAFE, CONICET-UBA), CC 67, Suc. 28, 1428 Buenos Aires, Argentina; mateofa@iafe.uba.ar \\ Received 2018 July 12; revised 2018 October 11; accepted 2018 October 14; published 2018 December 10
}

\begin{abstract}
Intergalactic space is believed to contain nonzero magnetic fields (the Intergalactic Magnetic Field: IGMF), which at scales of megaparsecs would have intensities below $10^{-9} \mathrm{G}$. Very high energy (VHE > $100 \mathrm{GeV}$ ) gamma-rays coming from blazars can produce $\mathrm{e}^{+} \mathrm{e}^{-}$pairs when interacting with the extragalactic background light (EBL) and the cosmic microwave background, generating an electromagnetic cascade of megaparsec scale. The IGMF may produce a detectable broadening of the emission beam that could lead to important constrains both on the IGMF intensity and its coherence length. Using the Monte Carlo-based Elmag code, we simulate the electromagnetic cascade corresponding to two detected TeV sources: PKS 2155-304 visible from the south and H1426+428 visible from the north. Assuming an EBL model and intrinsic spectral properties of the sources, we obtain the spectral and angular distribution of photons when they arrive at Earth. We include the response of the next generation Cherenkov telescopes by using simplified models for Cherenkov Telescope Array (CTA)-south and CTA-north based on a full simulation of each array performance. Combining the instrument properties with the simulated source fluxes, we calculate the telescope point-spread function for null and non-null IGMF intensities and develop a method to test the statistical feasibility of detecting IGMF imprints by comparing the resulting angular distributions. Our results show that for the analyzed source PKS 2155-304 corresponding to the southern site, CTA should be able to detect IGMF with intensities stronger than $10^{-14.5} \mathrm{G}$ within an observation time of $\sim 100 \mathrm{hr}$.
\end{abstract}

Key words: astroparticle physics - gamma rays: galaxies - magnetic fields

\section{Introduction}

Intergalactic space is believed to contain nonzero magnetic fields (the Intergalactic Magnetic Field: IGMF). It has been suggested that it could have originated in the early universe during the electroweak or QCD phase transition (Durrer \& Neronov 2013). A different proposed explanation suggests that this primordial magnetic field could have originated during the early formation of large-scale structures at redshifts $z \leqslant 10$ (Bertone et al. 2006). To date, we have no certain information about the IGMF intensity and spatial properties and there is no direct way of probing it with present techniques. However, in the last 10 years, several constrains to these parameters have been derived indirectly using different methods and techniques. The nonobservation of Faraday rotation induced by an IGMF in quasar observations suggests that its intensity is weaker than $10^{-9} \mathrm{G}$ for typical megaparsec-scale coherence lengths (Blasi et al. 1999; Pshirkov et al. 2015). Gamma-ray observations from distant active galactic nuclei (AGNs) allowed the estimation of lower limits and other constrains to the parameter space of the IGMF. An exclusion region in the range (0.3-3) $\times$ $10^{-15} \mathrm{G}$ was derived using HESS blazar TeV observations assuming a megaparsec-scale IGMF (Abramowski et al. 2014). Similarly, an exclusion region between $5.5 \times 10^{-15} \mathrm{G}$ and $7.4 \times 10^{-14} \mathrm{G}$ was calculated by VERITAS also using blazar $\mathrm{TeV}$ observations and megaparsec-scale coherence length assumptions (Archambault et al. 2017). Fermi-LAT observations in the $\mathrm{GeV}$ range also allowed the exclusion of fields below $\sim 10^{-19} \mathrm{G}$ for coherent lengths of $>1 \mathrm{Mpc}$ (Finke et al. 2015) and below $3 \times 10^{-16} \mathrm{G}$ for coherence lengths of $\geqslant 10 \mathrm{kpc}$ Ackermann et al. (2018).

Another study conducted by Arlen et al. (2014) makes a revision of other publications where they use methods to derive lower limits, and claims that a zero-IGMF hypothesis cannot be discarded with the available data. There is still a wide range of possible values for the spatial properties and intensity of the IGMF, gamma-ray interactions in the intergalactic medium could help constrain this parameter space and even detect the IGMF indirectly.

The universe is opaque for gamma-rays in the very high energy (VHE; >100 GeV) range. Photon absorption in the intergalactic (IG) photon backgrounds is energy dependent and starts to become substantial at TeV energies (Gould \& Schréder 1966). In particular, VHE gamma-rays from jets of AGNs can interact with photons in the IR-UV range present in the extragalactic background light (EBL) and photons from the cosmic microwave background (CMB), producing electronpositron pairs. These pairs carry most of the energy from the original photons, and can also interact with IG photons from the backgrounds via inverse Compton, promoting them to energies in the $\mathrm{HE}(>100 \mathrm{MeV})$-VHE range, and making them capable to pair produce in the IG backgrounds again. This cascade process converts the initial VHE photons into photons of lower energies, which can travel further. Moreover, depending on the intensity $(B)$ of the IGMF, the bending effect on the electron-positron pair trajectories can result in different emission scenarios. For a strong IGMF intensity $\left(B>10^{-7} \mathrm{G}\right)$ synchrotron cooling would become dominant and no secondary gamma-rays would be produced (Gould \& Rephaeli 1978); however, as mentioned above, this scenario has been ruled out for megaparsec-scale IGMF by the nonobservation of Faraday rotation. For a moderate IGMF $\left(10^{-12} \mathrm{G}<B<10^{-7} \mathrm{G}\right)$ the electron and positron pair trajectories are isotropized around the source eventually giving rise to an extended isotropic emission of photons, or halo, which take much longer to reach the observer than the direct photons from the source (Aharonian et al. 1994). For a weak IGMF $\left(B<10^{-14} \mathrm{G}\right)$, the cascade develops almost exclusively in the forward direction, although there is a broadening of the original emission beam, even for very small IGMF intensities. 
The extension of this emission depends on the IGMF intensity, its coherence length, and the source distance and should be clearly distinguished from the halo emission because in this case the broadening takes place along the jet direction, not in an isotropic way (Ahlers 2011; Abramowski et al. 2014). Different assumptions for the coherence length are present in the literature, ranging from $10^{-4}$ to $10^{4} \mathrm{Mpc}$. The general trend is that for relatively low coherent lengths $<1 \mathrm{Mpc}$, weak and moderate $\left(<10^{-15} \mathrm{G}\right)$ IGMF intensities are ruled out. This is a result of the random change in direction of the $\mathrm{e}^{+-}$pairs as they cross multiple coherent lengths. For relatively high coherent lengths $>1 \mathrm{Mpc}$, the intensity and coherence length are practically independent and almost all IGMF intensity scenarios are allowed (Finke et al. 2015). Assumptions on the Doppler factor $(\Gamma)$ and the opening angle associated to the emission jet may also play an important role in dimming or enhancing the resulting secondary radiation. Although some important effects are expected in the HE part of the energy spectrum for relatively high $\Gamma$ values $\left(\sim 10^{4}-10^{5}\right)$, for reasonably low values of $\Gamma<100$, effects in the VHE part of the spectrum can be considered negligible (Arlen et al. 2014).

Since this effect was proposed, several groups have tried unsuccessfully to observe it in the TeV band using multiple methods: Aharonian et al. (2001), Aleksić et al. (2010), Fallon (2010), Fernandez Alonso (2014), Abramowski et al. (2014), and Caprini \& Gabici (2015). Other authors found evidence of extended emission around extragalactic sources in the $\mathrm{GeV}$ range using Fermi-LAT observations (Chen et al. 2015; Kotelnikov et al. 2015). This extension could be potentially caused by the IGMF. All these studies were done using blazars, a subtype of AGNs that have their jets pointing toward the Earth and are therefore extremely luminous objects in the TeV band, perfect candidates to perform this type of studies. Since IGMF presence in the intergalactic medium will presumably affect the observed spectral and angular distributions of gamma-rays coming from blazars, methods usually consist of putting these distributions under a thorough analysis (Neronov \& Semikoz 2009; Aharonian et al. 2010).

The intergalactic cascade process is usually understood under the assumption that inverse Compton is the primary mechanism for the energy loss of the charged particles within the cascade. In their study, Broderick et al. (2012) question this idea and suggest that for bright sources $\left(\geqslant 10^{42} \mathrm{erg} \mathrm{s}^{-1}\right)$, plasma instabilities could be the main mechanism for energy loss of the produced pairs. In that case, the energy of the pairs would end up heating the intergalactic medium instead of scattering CMB or EBL photons, and no cascade process is produced.

In this work, we use MC based simulations of intergalactic cascades under different IGMF scenarios, and quantitatively study the effects of the magnetic field on the resulting spectral and angular distributions of the arriving photons. Motivated by the existence of a real future Cherenkov telescope system, the Cherenkov Telescope Array (CTA; Acharya et al. 2013), we assume a simplified model of response for CTA-south and CTAnorth and develop a method for testing the feasibility of detecting an extended component within the angular distribution of photons for each IGMF scenario. The resulting method constitutes an alternative way of studying the IGMF with the next generation Cherenkov telescopes, different from previous approaches usually based on possible IGMF imprints in the spectral energy distributions (SEDs) of VHE sources (Meyer et al. 2016). A
Table 1

Parameters of the Fitted Effective Area for Both Sites

\begin{tabular}{lccc}
\hline \hline Site & $A\left(\mathrm{~m}^{2}\right)$ & $B$ & $C$ \\
\hline South & $4.36 \times 10^{6}$ & 6.05 & 0.399 \\
North & $8.9 \times 10^{5}$ & 1.97 & 0.326 \\
\hline
\end{tabular}

preliminary discussion about the basis of this method was presented in Fernandez Alonso et al. (2015).

\section{Instrument Response}

Current imaging atmospheric Cherenkov telescopes (IACT) like HESS, MAGIC, and VERITAS have not been able to positively detect IGMF effects in TeV observations yet. The IGMF imprints are in principle present in both the spectral energy and the angular distributions, but are estimated to be relatively small. Instruments capable of detecting femto-Gauss IGMF effects are expected to have a considerable improvement on sensitivity as well as angular resolution in comparison with present instruments. CTA represents the next generation of Cherenkov Telecopes and will consist of two arrays covering a wider collection area and energy range than ever before for this kind of instruments. CTA is designed to increase significantly the effective area and the angular resolution as well as to improve the sensitivity in about an order of magnitude in relation to present instruments, making it a promising instrument for studying extended emission in the near future (Acharya et al. 2013). For this study, we consider a simplified model for the CTA array performance described in Ambrogi et al. (2016) and combine it with CTA-north and CTA-south public performance files to model each array separately (CTA 2017). In particular, we use analytic descriptions for the effective area, angular resolution, and point-spread function (PSF) shape to estimate the possible response of the telescopes to blazar observations.

\subsection{Effective Area and Sensitivity}

The effective area of an array of Cherenkov telescopes is determined by the total geometrical area covered by the array and the energy of the gamma-ray photon generating the light pool (Aharonian et al. 1997). The effective area for one possible CTA layout can be parameterized between $50 \mathrm{GeV}$ and $100 \mathrm{TeV}$ by the following expression (Ambrogi et al. 2016),

$$
A_{\text {eff }}(x)=\frac{A}{1+B \exp \left(-\frac{x}{C}\right)} .
$$

Here $x=\log (E / \mathrm{TeV})$, where $E$ is the gamma-ray energy. The corresponding parameters for the southern and northern sites are shown in Table 1.

Figure 1 shows the considered effective area as a function of energy for a possible CTA-south array and for the HESS telescope array (Vincent \& H.E.S.S. Collaboration 2004). It can be seen from the figure that the effective area for CTA increases significantly compared to the one corresponding to HESS, especially for energies above $1 \mathrm{TeV}$. Such an improvement in the collection area also implies improving the telescope sensitivity.

Sensitivity is defined as the minimum flux of gamma-rays required for a statistically significant detection in a given time. 


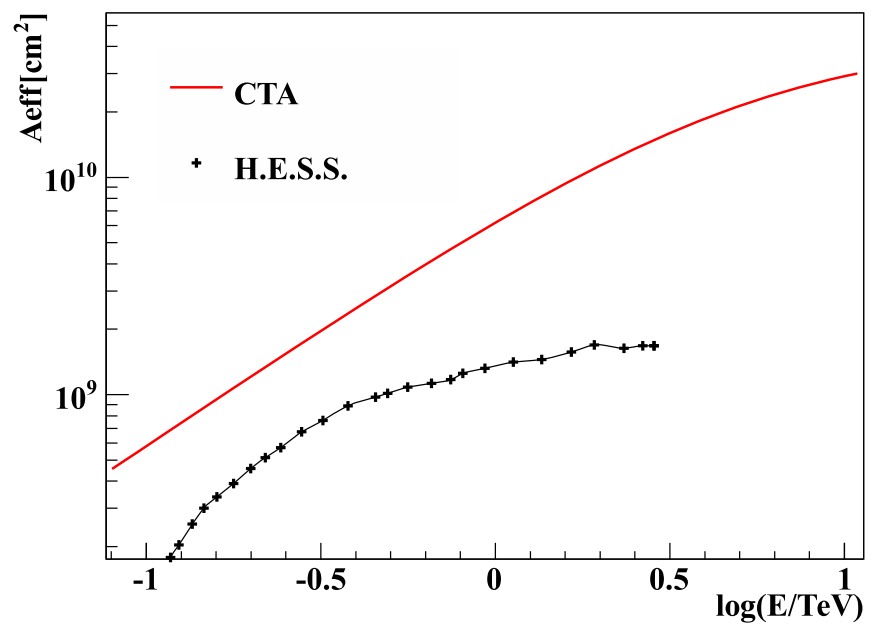

Figure 1. Effective area of CTA-South along with HESS effective area.

This is of course energy dependent and, in the case of current IACT, the energy boundaries for this flux lie between a few hundred $\mathrm{GeV}$ and $50 \mathrm{TeV}$. With the inclusion of four $23 \mathrm{~m}$ diameter Large Sized Telescopes (LST), CTA-south will lower the energy threshold down to $\sim 30 \mathrm{GeV}$ (see Figure 2), making it the first ground-based telescope to significantly detect photons with energies in this range. An improved sensitivity and a lower energy threshold may increase chances of detecting an extended component, with lower energy photons being the most affected, in principle, by the IGMF. However, as we will see in the next section, lowering the energy threshold is not necessarily always beneficial as there are other factors to be considered.

\subsection{Angular Resolution and PSF}

The telescope angular resolution is probably the most relevant instrumental property for this study, as it is based on the discrimination between point-like and non-point-like angular distributions. Here we consider CTA angular resolution $\left(\sigma_{\mathrm{PSF}}\right)$ proposed in Ambrogi et al. (2016), which is described by the following expression,

$$
\sigma_{\mathrm{PSF}}(x)=\alpha\left[1+\exp \left(-\frac{x}{\beta}\right)\right],
$$

where parameter $\alpha$ represents the achievable best resolution and parameter $\beta$ describes how fast the angular resolution changes with energy (Ambrogi et al. 2016). The best-fit parameters for the southern and northern sites are shown in Table 2. Given the IACT techniques for determining the gamma-ray direction, higher energy gamma-rays have more accurate direction determination than lower energy gammarays. For this reason, the considered energy threshold for the analysis is critical, since it will ultimately determine the angular resolution of the instrument.

The PSF, describes the angular response of the instrument to a point source, namely, the distribution of the angle $(\theta)$ formed by the reconstructed photon arrival direction and the direction of the source. This function depends on the angular resolution, which varies with the energy of the photon. The shape of this function, $f_{\mathrm{psf}}$, can be described by a simple Gaussian function

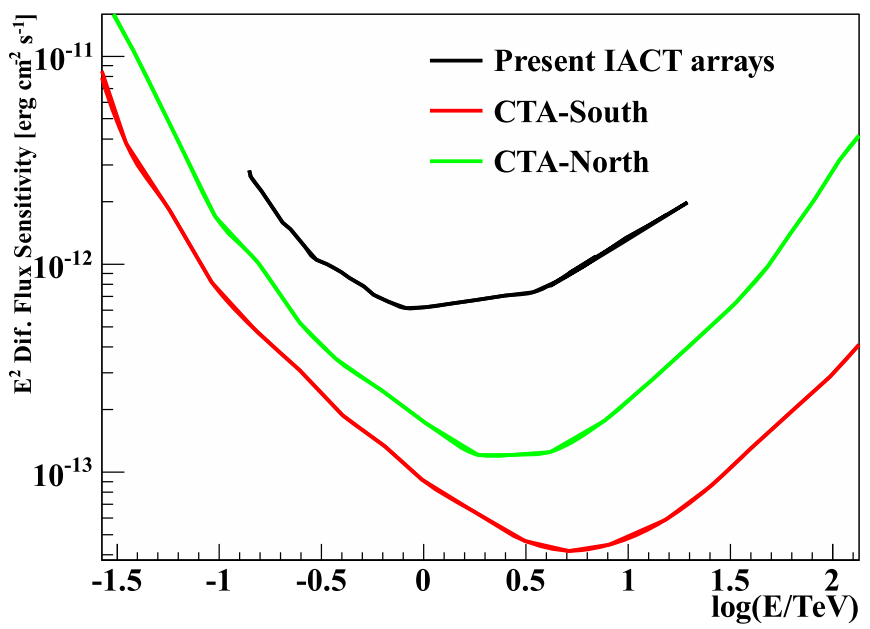

Figure 2. Sensitivity model for CTA-South (red line) and CTA-North (green line) taken from Acharya et al. (2013) and sensitivity for a present telescope array (black). All curves correspond to a $50 \mathrm{hr}$ observation time.

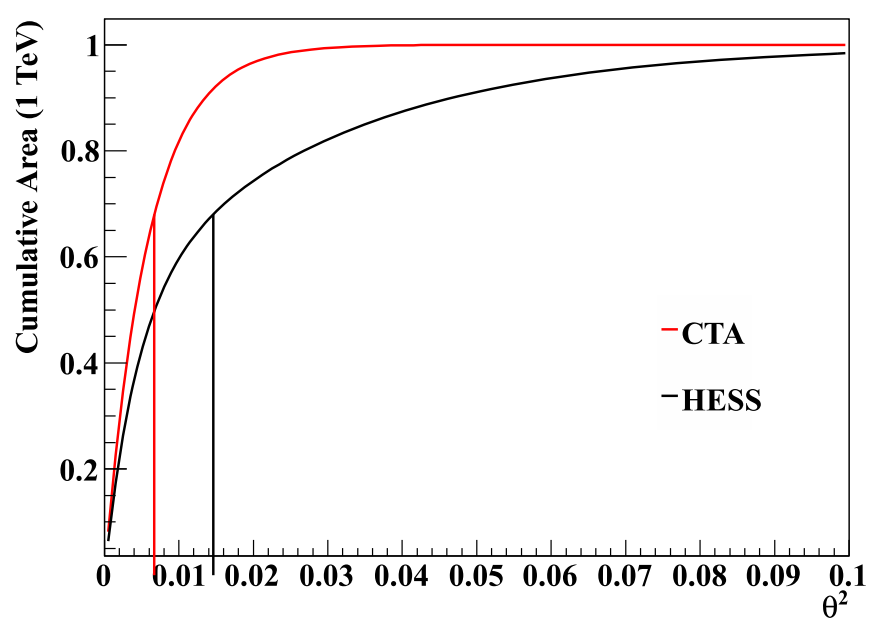

Figure 3. Normalized PSF cumulative distributions for CTA-south and HESS Vertical lines represent the values of $\theta^{2}$ that encloses $68 \%$ of the PSF area in each case.

Table 2

Parameters of the Fitted Angular Resolution for Both Sites

\begin{tabular}{lcc}
\hline \hline Site & $\alpha\left(^{\circ}\right)$ & $\beta$ \\
\hline South & 0.271 & 0.790 \\
North & 0.291 & 0.763 \\
\hline
\end{tabular}

(Ambrogi et al. 2016),

$$
f_{\mathrm{psf}}\left(\theta^{2}\right)=\exp \left(-\frac{\theta^{2}}{2 \sigma_{\mathrm{psf}}^{2}}\right)
$$

In this way, $\sigma_{\mathrm{psf}}$ determines the $68 \%$ containment radius of events $\left(\sigma_{68 \%}^{2}=-2 \ln (0.32) \sigma_{\mathrm{psf}}^{2}\right)$ and that defines the angular resolution of the instrument considered for this study. Figure 3 shows the normalized PSF cumulative area distributions for CTA-south (Ambrogi et al. 2016) and HESS (Aharonian \& Akhperjanian 2006) at $1 \mathrm{TeV}$. Vertical lines represent the value of $\theta^{2}$ that encloses $68 \%$ area of each PSF function; for this 
Table 3

Parameters for the Background Rate Function for the Southern and Northern Sites

\begin{tabular}{lcccccc}
\hline \hline Site & $A_{1}\left(\mathrm{~Hz} / \mathrm{deg}^{2}\right)$ & $\mu_{1}$ & $\sigma_{1}$ & $A_{2}\left(\mathrm{~Hz} / \mathrm{deg}^{2}\right)$ & $\mu_{2}$ & $\sigma_{2}$ \\
\hline South & 0.38 & -1.25 & 0.226 & 27.4 & -3.90 & 0.998 \\
North & 1.04 & -1.96 & 0.539 & $-2.83 \times 10^{4}$ & -10.4 & 0.114 \\
\hline
\end{tabular}

energy, CTA angular resolution drops down to below half the angular resolution achieved with the HESS telescope.

\subsection{Background Rate}

Cosmic rays constitute the main background for groundbased Cherenkov telescopes. This background is isotropic and has an approximately flat distribution in $\theta^{2}$. In this way, the background flux will unavoidably mix with the potential extended component of the source and the ability to distinguish this component will be directly affected by the background level. Present selection techniques allow us to discriminate between gamma-ray and cosmic-ray events quite efficiently; however, a fraction of these events cannot be distinguished and are detected as gamma-rays. The reason for this is that these selection techniques are usually based on the differences between the images of showers produced by cosmic- and gamma-rays, and these differences are not always clear, specially as the energy of the primary particle drops and the image reconstruction gets less accurate. The background rate of the instrument is the number of cosmic-ray events that are systematically mistaken with gamma-ray events per unit of time. Having a good estimate of this rate allows us to extract the right amount of background flux from the desired signal. For this study, we use the background rate per unit of solid angle proposed in Ambrogi et al. (2016), that is energy dependent and it is described by the following expression,

$$
\begin{aligned}
B_{r}(x)= & A_{1} \exp \left(-\frac{\left(x-\mu_{1}\right)^{2}}{2 \sigma_{1}^{2}}\right) \\
& +A_{2} \exp \left(-\frac{\left(x-\mu_{2}\right)^{2}}{2 \sigma_{2}^{2}}\right)+C .
\end{aligned}
$$

The resulting parameters for the southern and northern sites are displayed in Table 3. The background rate decreases with energy as expected. The overall background level is obtained by integrating this function between a chosen low energy threshold and $30 \mathrm{TeV}$. It should be noted that lowering the energy threshold means increasing the background level, this is an important fact to be considered when choosing the threshold for this and other analyses as well.

\section{Source Selection}

There are a number of factors that make a particular source more likely to present a significant extended flux component than others. Distance/redshift is a crucial property: if the source is too close, the cascade process may not have enough distance to develop an appreciable broadening. If the source is too distant, the overall flux may be too low and the effect, although existent, will be too dimmed to be detected. Intrinsic spectral index is also a factor that could be determinant, since VHE gamma-rays will produce more energetic and richer cascades than lower energy photons, "hard" spectrum sources are more likely to present an appreciable extended flux

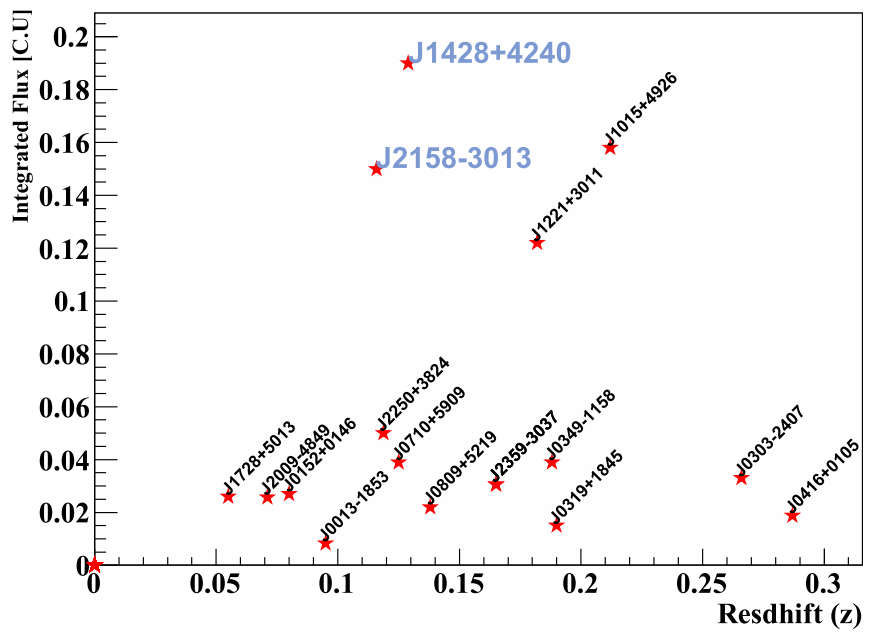

Figure 4. Integrated flux vs. redshift of a sample of 17 detected HBLs. Selected sources are highlighted in light blue.

component. Blazars are by far the brightest extragalactic $\mathrm{TeV}$ sources, HBL subtype being the most numerous among the detected $\mathrm{TeV}$ sources. There are about 50 detected HBLs in the TeVCat catalog (Wakely \& Horan 2008), presenting a variety of redshifts and fluxes. Figure 4 shows a sample of 17 HBLs with good redshift and flux determination. Flux and redshift data were taken from Wakely \& Horan (2008) and Carosi et al. (2016). For this study, we considered two different blazars: PKS 2155-304 visible from the southern hemisphere and $\mathrm{H} 1426+428$ visible from the northern hemisphere, which combine, in principle, good properties for developing an extended component. The corresponding $\mathrm{TeV}$ names for these sources as shown in Figure 4 are TeV-J2158-3013 and TeV$\mathrm{J} 1428+4240$. Table 4 shows a summary of the relevant parameters considered in this study for the selected sources.

Due to gamma-ray absorption and the cascade process taking place in the intergalactic medium, the observed spectral index is expected to be higher than the intrinsic one. The absorption process depends on the EBL properties and, although there are models that could be used to estimate the source intrinsic spectrum, EBL properties are rather uncertain. On the other hand, photons in the $\mathrm{GeV}$ range suffer almost no absorption and the observed spectral index should not differ too much with the intrinsic one at these energies. Following this argument, we consider the $\mathrm{TeV}$ part of the intrinsic spectra of the sources to be a prolongation of the $\mathrm{GeV}$ part. Therefore, the intrinsic spectrum should be well described by a power law with the observed GeV spectral index reported in the Fermi-LAT 4Year Point Source Catalog (Acero et al. 2015). An exponential cutoff was set in $10 \mathrm{TeV}$ to ensure a sufficient amount of VHE gamma-rays (Eungwanichayapant \& Aharonian 2009).

$$
\frac{d N}{d E}(E)=N_{0} E^{-\Gamma} \exp (-E / 10 \mathrm{TeV}),
$$

where $N_{0}$ is a normalization constant. 
Table 4

Considered Sources and Their Relevant Parameters

\begin{tabular}{|c|c|c|c|}
\hline Source & Redshift & Spectral Index $\Gamma$ & Integrated Flux $\left(\mathrm{cm}^{-2} \mathrm{~s}^{-1}\right)-\mathrm{CU}^{\mathrm{a}}$ \\
\hline PKS 2155-304 & 0.11 & 1.83 & $1.9 \times 10^{-11}-0.15$ \\
\hline H $1428+428$ & 0.129 & 1.57 & $2.53 \times 10^{-11}-0.2$ \\
\hline
\end{tabular}

Note.

${ }^{\mathrm{a}} \mathrm{CU}=1.27 \times 10^{-11} \mathrm{~cm}^{-2} \mathrm{~s}^{-1}$ obtained by integrating Crab differential spectrum between 0.3 and $30 \mathrm{TeV}$.

Flux variability is also an important factor to be considered. Blazars are especially known for their flux variability in the $\mathrm{GeV}-\mathrm{TeV}$ range, presenting periods of high emission better known as flares. During these periods, flux can rapidly increase up to several orders of magnitude with respect to the typical or quiescent flux of the source, and normal spectral properties of the source change, usually hardening the spectral index. As a result, a possible broadening effect in the emission can be outshined by the direct emission coming from the source. For this reason, the data sets used to study magnetic broadening are usually discriminated in those coming from high activity and low activity periods. In particular, the source PKS 2155-304 has reported a relatively high variability index (Acero et al. 2015), so the spectral properties considered in this study were taken from observations of the source during a quiescent state (H.E.S.S. Collaboration et al. 2010). Possible effects from past flares could, in principle, be present in the quiescent spectrum as well; these effects are commonly referred to in the literature as pair echoes (Takahashi et al. 2011). Although pair echoes are not contemplated in this study, their possible effects would at least positively contribute to the broadening, so our considered scenarios should be pessimistic regarding this point.

\section{Simulations}

Intergalactic electromagnetic cascades are simulated using the Monte Carlo-based code Elmag (Kachelrieß et al. 2012) version 2.03. The simulation injects photons (or leptons) into the intergalactic space at a chosen redshift, and takes into account pair production interactions with the EBL and the $\mathrm{CMB}$, inverse Compton interactions of the resulting pairs and their synchrotron losses, and deflections in the IGMF. Input settings include an EBL model, IGMF coherence length and intensity, and the source injection spectrum. The simulation generates the spectral and angular distributions of photons when they arrive at Earth. The method presented in this work is strongly based on the simulated direction and energy of the arriving photons, so a few comments on how these are obtained and treated are necessary. The simulation assumes a small deflection angle approximation, for strong IGMF intensities $\left(B>1 \times 10^{-14} \mathrm{G}\right)$ where deflections start to be considerably large, especially for lower energetic particles, the program emulates an isotropic emission scenario by randomly assigning a direction to the scattered photons. For isotropically emitted photons, only those whose direction lies within the jet cone are considered, those scattered with bigger angles than the jet angle are dismissed. As a result, strong IGMF scenarios tend to show SED with a less populated low energy part.

For this study, we simulate 10 samples of $5 \times 10^{5}$ photons, which are injected by the source following a power-law intrinsic spectrum, as described by Equation (5). Each sample is simulated with a different random initialization number to account for fluctuations coming from the MC process. The

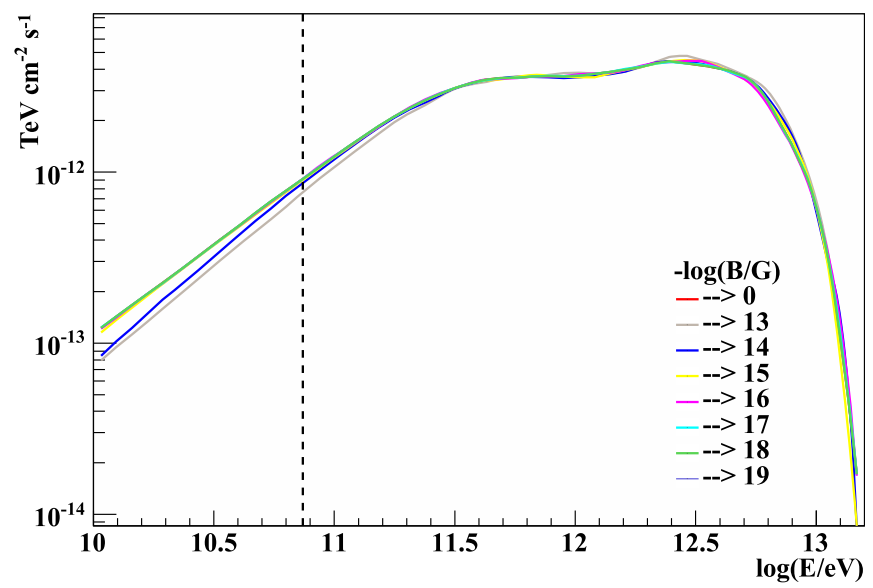

Figure 5. SED for different IGMF intensities considering the EBL model proposed in Domínguez et al. (2011). The dashed vertical line represents the lower energy threshold of $75 \mathrm{GeV}$ considered in this study.

samples are then stacked together to improve statistics as much as possible. We assume the EBL model proposed in Domínguez et al. (2011) and an IGMF coherence length of $1 \mathrm{Mpc}$. A thinning factor of 0.3 is applied to speed up calculations. All simulations were done on an IGMF intensity grid within the range $\log (\mathrm{B} / \mathrm{G})=-19$ to $\log (\mathrm{B} / \mathrm{G})=-14$, including a null field $(B=0 \mathrm{G})$, which is used as a nonexistent IGMF scenario. Stronger fields $\left(>10^{-14} \mathrm{G}\right)$ were not considered in order to avoid conflict with the small angle approximation. Alternatively, we consider a different EBL scenario described by Gilmore et al. (2012) to study possible effects introduced by differences in the absorption process. The Gilmore et al. (2012) model is chosen because it presents considerable differences in the spectral shape in relation to the Domínguez et al. (2011) model, namely, a lower radiation density in the far-IR region. Finally, for the source PKS 2155-304, two alternative spectral indexes are explored to study the effect of spectral differences in the output angular distributions. Sources that present a harder spectrum inject more high energy photons into the IG medium and are expected to produce a higher cascade component than soft sources. Two values are chosen, corresponding to a variation of $35 \%$ up and down from the spectral index value reported in the Fermi-LAT 4-Year Point Source Catalog, i.e., $\Gamma=1.35$ and $\Gamma=2.30$.

\section{Method}

As mentioned before, there are several possible ways for searching IGMF imprints. The SED would in principle contain IGMF traces, particularly in the lower energy range, where the direction of the last scattering photons is determined by the electron-positron pairs that are more sensitive to the magnetic field strength. Figure 5 shows SEDs for PKS 2155-304 simulated for different IGMF intensities. Spectra are well 
distinguished from each other in the lower energy range, especially for relatively high IGMF intensity scenarios. As the intensity weakens and the energy increases, spectra become indistinguishable from each other. In fact, above energies around $75 \mathrm{GeV}$, represented by the vertical dashed line in Figure 5, SEDs are practically indistinguishable for any IGMF scenario with $B \leqslant 10^{-14}$. On the other hand, the angular distribution of photons is also expected to contain IGMF traces and could potentially lead to constraints or even a detection. Any significant difference between a source angular distribution and the one corresponding to a point source, i.e., the telescope PSF, would indicate the existence of an extended contribution. Our strategy consists of comparing simulated distributions of nonzero IGMF scenarios with a point-like distribution constructed from a zero-IGMF simulation; we test the power of a CTA-like instrument to distinguish the angular distributions of those two simulations. More specifically we test, for a given IGMF scenario, the probability of rejecting the null hypothesis in which nonzero and null IGMF $\theta^{2}$ distributions come from the same parent distribution.

\subsection{PSF Convolution}

To obtain a realistic representation of the observed angular distributions, the output of the simulated events are convolved with the telescope PSF. The convolution process is done by getting the energy of each simulated event and randomly sampling Equation (3) evaluated in that energy. The resulting value of $\theta_{\mathrm{psf}}^{2}$ is then used to obtain the reconstructed arrival direction of the photon, $\theta^{2}$, by using the following expression,

$$
\theta^{2}=\theta_{\mathrm{sim}}^{2}+\theta_{\mathrm{psf}}^{2}+2 \theta_{\mathrm{sim}} \theta_{\mathrm{psf}} \cos \phi,
$$

where $\theta_{\text {sim }}^{2}$ is obtained from the Elmag simulation and $\phi$ (azimuth angle) is uniformly sampled from the interval $(0,2 \pi]$ to account for the symmetry under azimuthal rotation. Each event is also weighted considering the telescope effective area corresponding to the event energy. Finally, an overall factor is applied to normalize the total number of events according to the source integrated flux (see Table 4), which is obtained by integrating the observed spectrum of each source, and a given observation time.

\subsection{Fluctuations and Background Subtraction}

Fluctuations and background events are added to the convolved simulated distribution corresponding to an IGMF of $B=0$. To achieve this, each bin content $\mu_{i}$ of the $\theta^{2}$ histogram is used to sample a Poisson distribution, which is given by,

$$
P(n ; \mu)=\frac{\mu^{n}}{n !} e^{-\mu},
$$

where $\mu=\mu_{i}$ for the $i$ th bin. The sampled value, $n_{i}$, is then used to construct a new fluctuated distribution $H_{\text {fluct }}\left(\theta^{2}\right)$, where each bin content is a different Poisson-fluctuated value of the original bin content. Additionally, the average number of background events is obtained by integrating the background rate given by Equation (4) between the corresponding lower energy threshold and $30 \mathrm{TeV}$,

$$
\mu_{b}=\pi T_{\mathrm{obs}} \Delta \theta \int_{E_{\mathrm{th}}}^{30 \mathrm{TeV}} B_{r}(E) d E
$$

where $B_{e}$ is the background rate, $T_{\mathrm{obs}}$ is the observation time, and $\Delta \theta$ is the bin width of the histogram under consideration. The resulting value $\mu_{b}$ is then used to sample a Poisson distribution (Equation (7) with $\mu=\mu_{b}$ ). The sampled background events are added bin-wise to the $H_{\text {fluct }}\left(\theta^{2}\right)$ distribution to obtain a raw observed distribution $H_{\text {raw }}\left(\theta^{2}\right)$. Finally, the background is subtracted following the Wobble method (Finnegan 2011), where three different patches corresponding to the off-source region of the sky are simultaneously observed along the on-source region, and then used for background subtraction. This procedure was emulated by sampling a Poisson distribution with mean value $\mu_{W}=3 \mu_{b}$. The sampled value is then subtracted from each bin of the $H_{\text {raw }}\left(\theta^{2}\right)$ distribution to obtain the excess distribution $H_{\text {excess }}\left(\theta^{2}\right)$.

\subsection{Rejection}

Non-null magnetic field models, obtained in the same way as the PSF (see Section 5.1) but in this case without including either Poisson fluctuations or background, are then fitted to the excess distribution $\left(H_{\text {excess }}\right)$. The fitting parameter is a normalization constant that is obtained by minimizing the $\chi^{2}$. The analytic expression for the parameter is given by,

$$
A=\frac{\sum_{i}\left(H_{\text {excess }, \mathrm{i}} H_{\text {model }, i}\right) / \sigma_{i}^{2}}{\sum_{i} H_{\text {model }, i}^{2} / \sigma_{i}^{2}},
$$

where $H_{\text {excess,i }}$ and $H_{\text {model }, i}$ are the contents of the $i$ th bin for the excess and model distributions respectively, and $\sigma_{i}$ represents the standard deviation of the $i$ th bin of the excess distribution. By using the Poissonian character of all fluctuation the variance of $i$ th bin content can be estimated as,

$$
\sigma_{i}^{2}=H_{\mathrm{raw}, i}+\mu_{b, i}+\frac{\mu_{W, i}}{3} .
$$

Finally, a $\chi^{2}$ test is applied to obtain the $p$-value of the fit. This process is repeated 1000 times for every IGMF intensity, and each time with a different fluctuated excess distribution to emulate different possible observations. Our null hypothesis is to consider the excess PSF distribution $(B=0)$ and the simulated model (non-null magnetic field) as coming from the same parent distribution. The probability of rejecting this hypothesis with a $99 \%$ confidence level is then given by the number of cases whose $p$-value lies below 0.01 , over the total number of cases considered.

\section{Results and Discussion}

A preliminary study was performed to determine the optimal low energy threshold to be considered in the analysis. In previous sections, we discussed how lowering the energy threshold will not necessarily enhance the cascade effect in the overall distributions. Many of the telescope performance parameters, such as background level and angular resolution, will be inevitably affected negatively as the threshold energy decreases. To estimate the impact of the energy threshold we considered five different low threshold values: $30,50,75,150$, and $200 \mathrm{GeV}$ and calculated the rejection ratio for an IGMF intensity of $B=10^{-14} \mathrm{G}$ and for three different values of the observation time, 50, 100, and $150 \mathrm{hr}$. Figure 6 shows the 


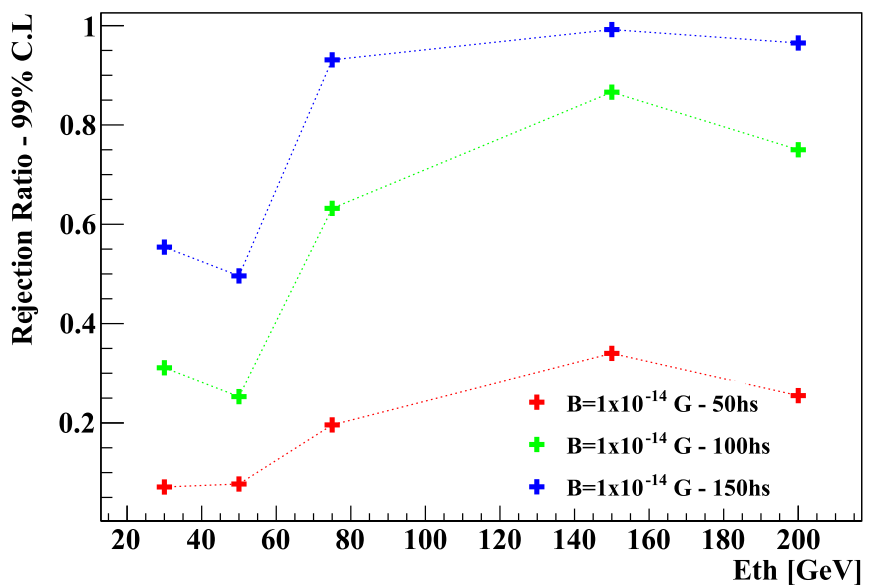

Figure 6. Rejection probabilities for PKS 2155-304 (CTA-south) for different low energy thresholds. The results correspond to the IGMF scenario with intensity $B=10^{-14} \mathrm{G}$.

Energy Threshold $=75 \mathrm{GeV}--\Gamma=1.83--$ EBL: Dominguez et al.

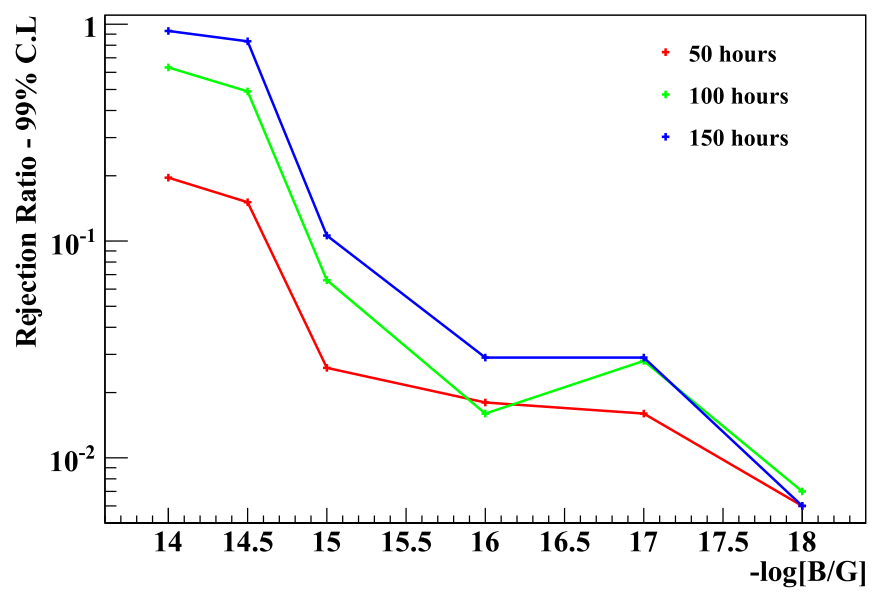

Figure 7. Rejection probabilities for PKS 2155-304 (CTA-south) for different observation time windows.

resulting rejection probabilities for each case. It is clear from this example that neither the lowest nor the highest energy thresholds present the best probabilities of detecting a possible broadening. It might be tempting to say that $150 \mathrm{GeV}$ is the optimal energy threshold, however, the results shown represent only the behavior of one particular source and one particular IGMF intensity. The rejection ratio trend with energy threshold is likely to depend on the source characteristics and even on the IGMF intensity. For these reasons, a more conservative $75 \mathrm{GeV}$ lower energy threshold is adopted throughout the rest of the analysis.

Figures 7 and 8 show the resulting rejection probabilities for PKS 2155-304 and H1426+428, respectively, as a function of the IGMF intensity and for a low energy threshold of $75 \mathrm{GeV}$ and for 50, 100, and $150 \mathrm{hr}$ of observation time. In general, the probability is considerably higher IGMF intensities above $\sim 10^{-14} \mathrm{G}$, where it increases with longer observation time windows. The rejection ratio then drops down for weaker magnetic fields in both the southern and northern sites; however, in the latter, the ratios are smaller and the drop is more abrupt, probably because of the differences in the instrument performance. The drop in the rejection ratio for

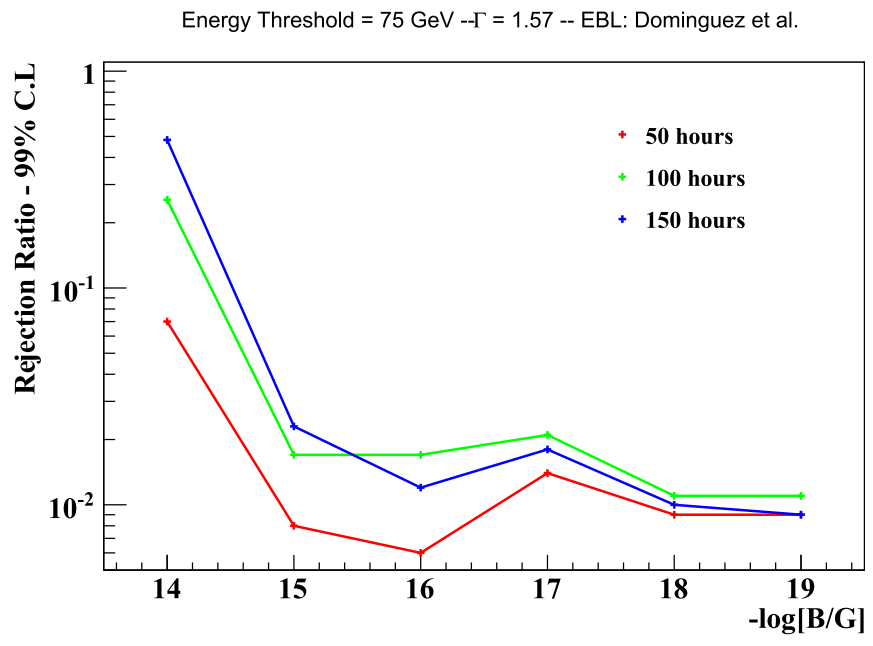

Figure 8. Rejection probabilities for H1426+428 (CTA-north) for different observation time windows.
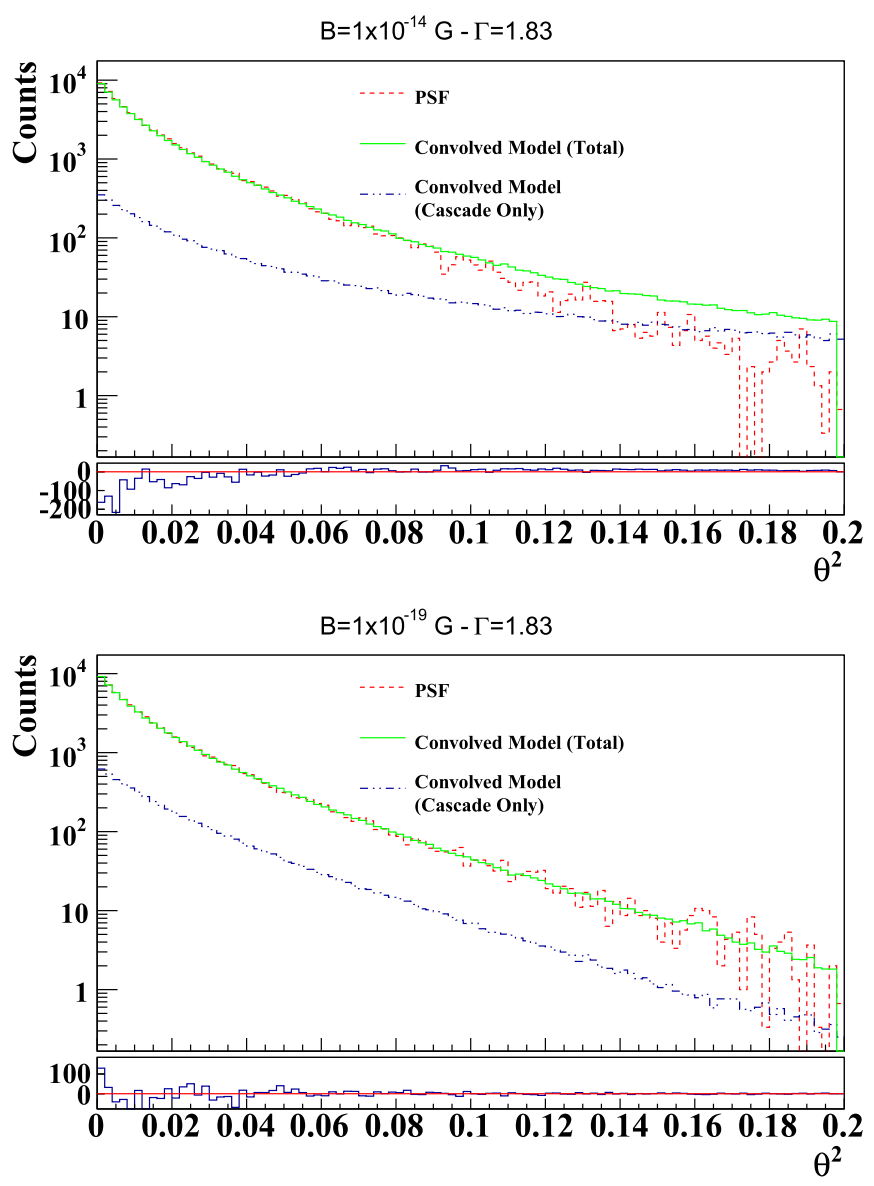

Figure 9. Fluctuated PSF $\theta^{2}$ distribution fitted with the $\theta^{2}$ distributions corresponding to $B=10^{-14} \mathrm{G}$ (top) and $B=10^{-19} \mathrm{G}$ (bottom). Also shown are the $\theta^{2}$ distributions corresponding to the cascade photons. Lower pads show the bin content difference between the convolved model $\theta^{2}$ distribution and the PSF distribution in each case.

weak magnetic fields responds to the fact that in these scenarios, the cascade component is too weak and/or not broadened enough to be differentiated from the PSF distribution and its fluctuations.

We have discussed how stronger IGMF scenarios lead to broader angular dispersion of the arriving photons, which can 

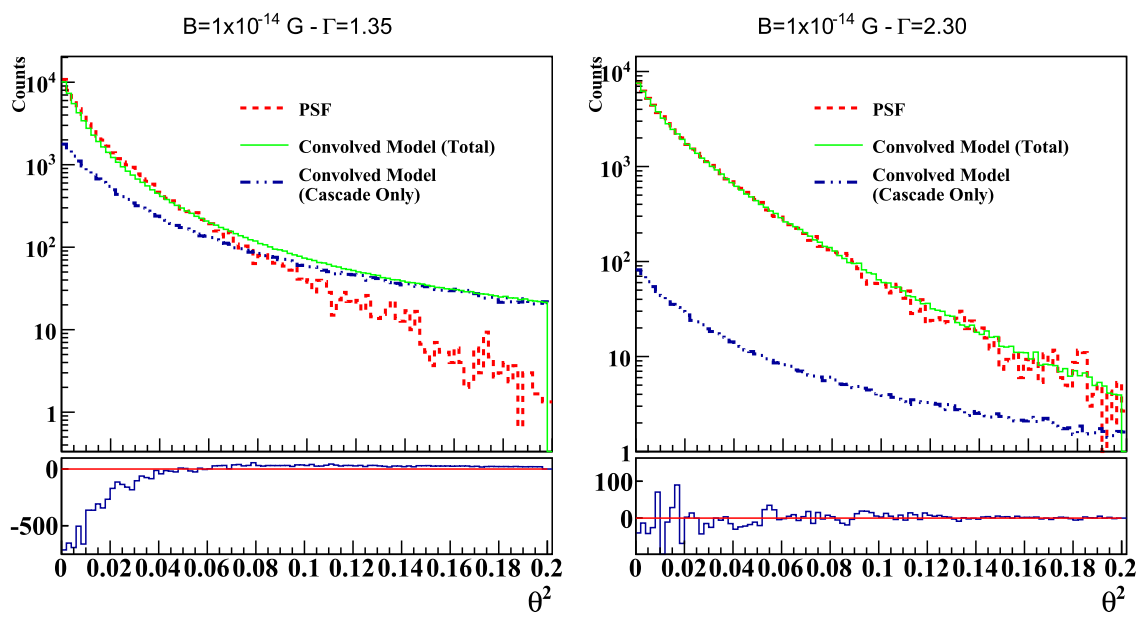

Figure 10. Fluctuated PSF $\theta^{2}$ distribution fitted with the $\theta^{2}$ distributions corresponding to $B=10^{-14} \mathrm{G}$ for spectral indexes $\Gamma=1.35$ (left) and $\Gamma=2.30$ (right). Also shown are the $\theta^{2}$ distributions corresponding to the cascade photons. Lower pads show the bin content difference between the convolved model $\theta^{2}$ distribution and the PSF distribution in each case.

be directly appreciated in the $\theta^{2}$ distributions of the arriving photons. Figure 9 shows the fits of PKS 2155-304 PSF, for two particular samples, with the $\theta^{2}$ distributions (after the PSF convolution) corresponding to two IGMF intensities, a strong field $\left(B=10^{-14} \mathrm{G}\right)$ and a weak field $\left(B=10^{-19} \mathrm{G}\right)$. Also shown is the $\theta^{2}$ distributions of the cascade photons. The convolved model $\theta^{2}$ distribution is clearly broader than the PSF distribution in the case of strong magnetic field, whereas for the weak magnetic field scenario these two distributions are almost indistinguishable. The amount of photons within the cascade distribution and its shape, will ultimately determine whether or not the broadening is appreciable.

The IGMF intensity is not the only factor that affects the shape and broadening of the cascade distribution. A less direct IGMF effect imprinted in the $\theta^{2}$ distribution comes from the energy spectrum of the photons emitted from the source. As discussed in Section 4, hard spectrum sources are expected to inject a higher amount of high energy photons capable of generating secondary gamma-rays and thus resulting in a relatively more important cascade component. Figure 10 shows the fits of PKS 2155-304 PSF, for two particular samples, with the $\theta^{2}$ distributions (after the PSF convolution) corresponding to $B=10^{-14} \mathrm{G}$ and for two alternative spectral indexes of $\Gamma=1.35$ and $\Gamma=2.30$. Also shown are the $\theta^{2}$ distributions of the cascade photons. The spectral index effect can be clearly appreciated in both the flux level and shape of the cascade distributions. The cascade component in the case of $\Gamma=1.35$ represents a significant part of the total flux, and the shape of its distribution clearly differs from the shape of the PSF. On the other hand, the $\theta^{2}$ distribution for $\Gamma=2.30$ has a much less significant cascade component and the overall distribution cannot be distinguished from the PSF and its fluctuations. Figure 11 shows the rejection probabilities for these last two cases with alternative spectral indexes. As expected, the hard spectrum case shows, in general, higher rejection ratios than the cases with softer spectral index. For the IGMF scenario with $B=10^{-14} \mathrm{G}$, the null hypothesis is rejected regardless of the observation time. The hard spectrum case also presents a smoother transition to weaker IGMF intensities, showing promising results for scenarios with intensities of $\sim 10^{-15} \mathrm{G}$. On the other hand, the softer spectral index shows small rejection ratios for all IGMF scenarios, reinforcing the idea that
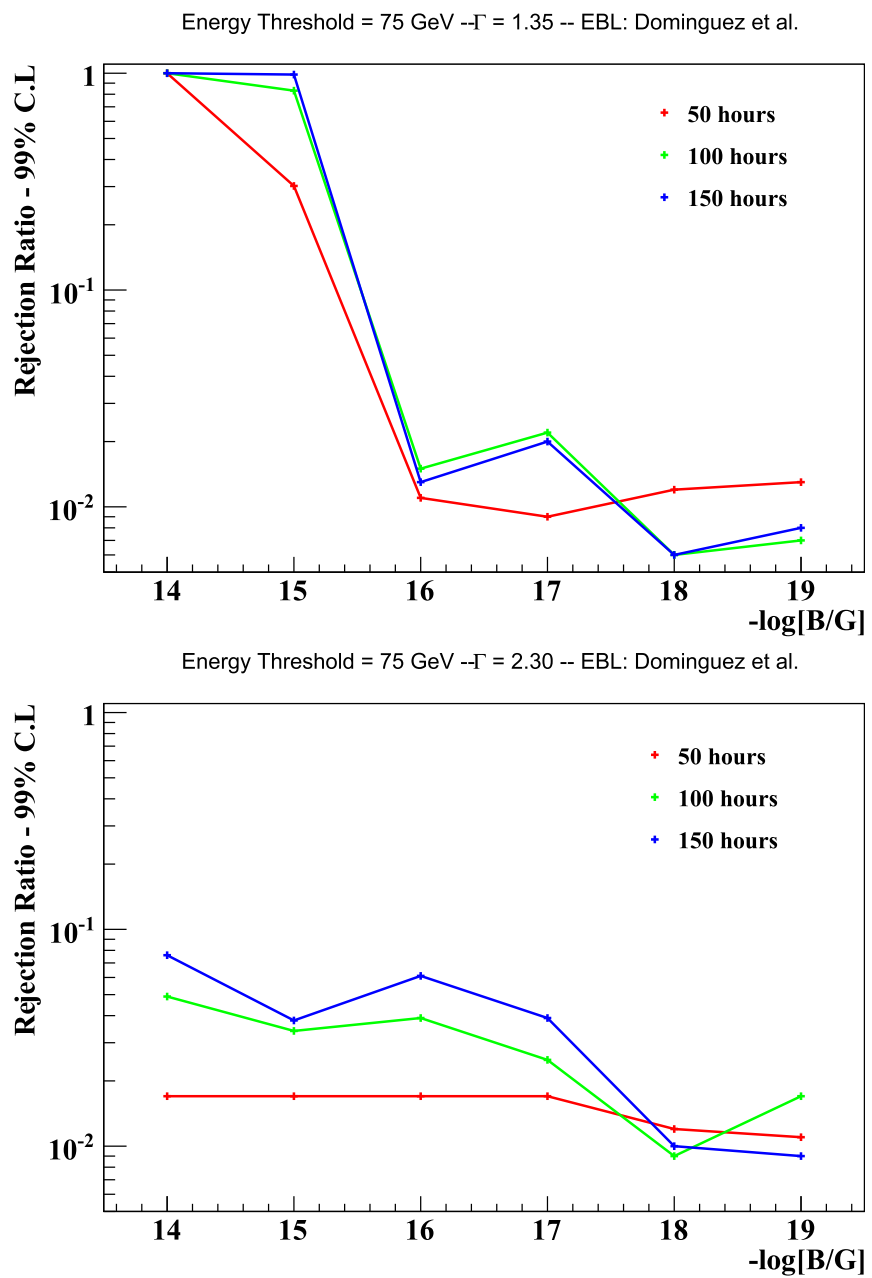

Figure 11. Rejection ratios for PKS 2155-304 (CTA-south) with alternative spectral indexes of $\Gamma=1.35$ (top) and $\Gamma=2.30$ (bottom).

the IGMF studies are less promising when soft spectrum sources are considered. Regarding the energy threshold discussed previously, the hard spectrum case shows an improvement on the rejection ratios as the energy threshold decreases. This can be attributed to the fact that most of the secondary photons that constitute the cascade component, 
Energy Threshold $=75 \mathrm{GeV}--\Gamma=1.83$-- EBL: Gilmore et al.

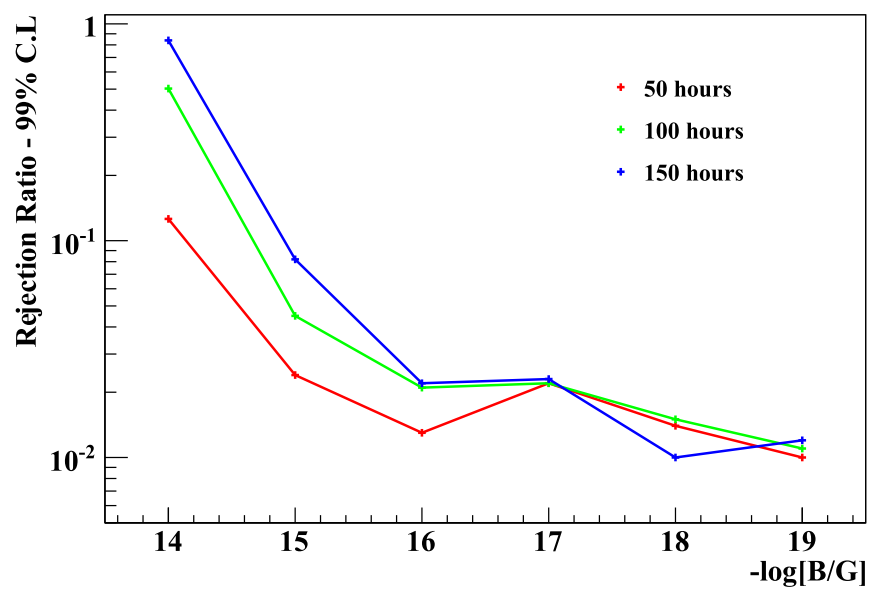

Figure 12. Rejection ratios for PKS 2155-304 (CTA-south) considering the EBL model proposed in Gilmore et al. (2012).

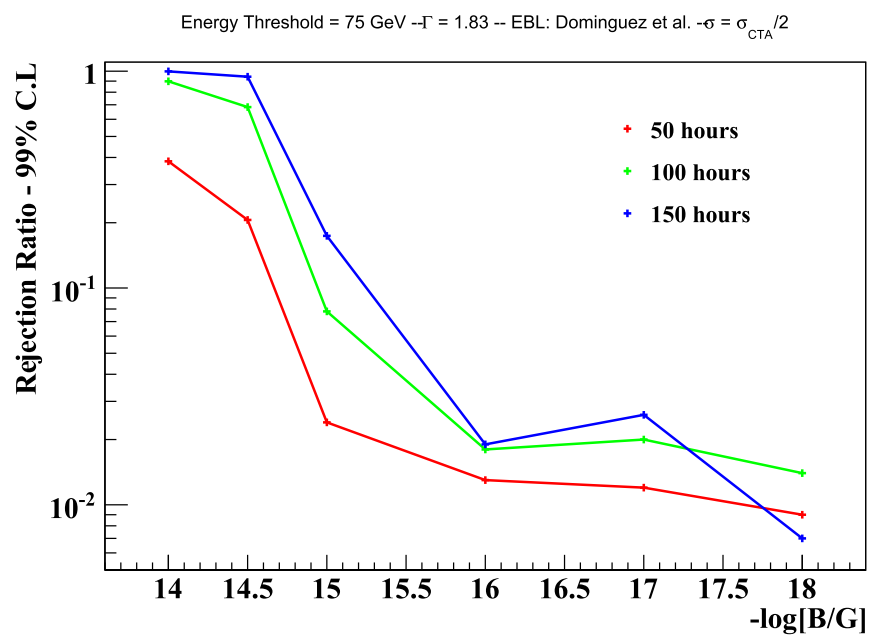

Figure 13. Rejection ratios for PKS 2155-304 (CTA-south) considering a narrower PSF with $\sigma_{\mathrm{psf}} / 2$.

populate the lower energy range of the SED, and being the case with most significant cascade component, it is expected that a lower cut in energy will result in an enhancement of the cascade distribution.

Figure 12 shows the rejection ratios corresponding to PKS 2155-304 when considering the EBL model proposed in Gilmore et al. (2012). Any difference in the absorption process that could be introduced by changing the EBL model are simply too small to be appreciated in the current $\theta^{2}$ distributions and the resulting rejection ratios are practically the same as the ones obtained with the Domínguez et al. (2011) EBL model.

Alternative intrinsic source spectra for PKS 2155-304 with energy cutoffs in $E_{\text {cut }}=5 \mathrm{TeV}$ and $E_{\text {cut }}=20 \mathrm{TeV}$ were considered to estimate the impact of high energy photons in the broadening effect. Results show that in the case of a $5 \mathrm{TeV}$ cutoff, rejection probabilities drop below 0.1 for all fields and observation times. This is consistent with the fact that, for this case, fewer high energy photons are being injected in the intergalactic medium, producing a less significant amount of cascade photons. On the other hand, a cutoff in $20 \mathrm{TeV}$ shows no significant changes in relation to the $10 \mathrm{TeV}$ cutoff spectrum, perhaps suggesting that, in spite of the higher cutoff, the amount of injected photons is not enough to produce detectable improvements in the rejection ratios. If this is the case, a plateau in the rejection ratios would be expected even for higher energy cutoffs.

To estimate the limitations due to the instrument performance, the rejection probabilities were also calculated considering a narrower PSF. This was achieved by halving the $68 \%$ containment radius of the PSF, $\sigma_{\mathrm{psf}}$ in Equation (3). Results for the improved instrument are shown in Figure 13.

Although probabilities for this case are slightly higher, pushing the detection limit toward weaker IGMF intensities, the values are still too low to claim any detection around $\sim 10^{-15} \mathrm{G}$. However, it is reasonable to expect higher values as the angular resolution of the instrument improves.

\section{Conclusions}

The possible magnetic broadening effect in the angular distribution of gamma-rays coming from distant blazars constitutes an alternative method to study and constrain the IGMF. The aim of this study is to try to asses quantitatively the detection of possible magnetic broadening with next generation Cherenkov telescopes, given a realistic set of observations and instrumental response. The detection will ultimately rely on which source or sources are chosen for the study and what method is used for discriminating the cascade component. For the analyzed source PKS 2155-304 corresponding to the southern site, results show that CTA should be able to detect IGMF with intensities stronger than $10^{-14.5} \mathrm{G}$ within an observation time of $\sim 100 \mathrm{hr}$. The source H1426+428 corresponding to the northern site shows a similar trend, although in this case rejection ratio values are lower, probably due to the instrumental limitations. The obtained results also give us some valuable information on what factors are especially determinant. Source spectral index and flux seem to be key properties. The source PKS 2155-304 shows significant changes when the spectral index is varied by $30 \%$, a reasonable amount given the uncertainties in this parameter. The soft version of its spectrum shows low rejection probabilities for all scenarios, whereas the hard one increases them and pushes the detection range down to IGMF intensities of $\sim 10^{-15} \mathrm{G}$. Spectral index and flux level should be prioritizing properties when looking for suitable source candidates. The instrument performance is also determinant. There is a clear difference in the results coming from the 50, 100, and $150 \mathrm{hr}$ observation times. In this sense, the effective area of the instrument will ultimately determine whether it is practical or not to expect positive results from typical observation times. Background fluctuations are also related to the observation time, and they are crucial when it comes to discriminating the extended component. Any improvement in the treatment of these fluctuations will certainly have a positive impact on the detection of the broadening. Finally, the method also relies on the angular resolution of the instrument, and although our method shows slightly better results for a PSF with half the width, it is expected that future experiments will significantly increase their chances as they improve their angular resolution.

This work has been partially supported with a grant from Agencia Nacional de Promoción Científica y Tecnológica, Argentina. The author M.F.A. developed this work with a Doctoral fellowship from CONICET, Argentina. The authors 
A.D.S. and A.C.R. are members of the Carrera del Investigador Científico of CONICET, Argentina. We thank the anonymous referee for a careful reading and useful questions and suggestions that improved the presentation of this paper.

\section{References}

Abramowski, A., Aharonian, F., Ait Benkhali, F., et al. 2014, A\&A, 562, A145 Acero, F., Ackermann, M., \& Ajello, M. 2015, yCat, 221

Acharya, B. S., Actis, M., Aghajani, T., et al. 2013, APh, 43, 3

Ackermann, M., Ajello, M., \& Baldini, L. 2018, ApJS, 237, 32

Aharonian, F., \& Akhperjanian, A. 2006, A\&A, 457, 899

Aharonian, F., Akhperjanian, A., Barrio, J., et al. 2001, A\&A, 366, 746

Aharonian, F., Coppi, P., \& Voelk, H. 1994, ApJL, 423, L5

Aharonian, F. A., Hofmann, W., Konopelko, A. K., \& Völk, H. J. 1997, APh, 6,369

Aharonian, F. A., Kelner, S. R., \& Prosekin, A. Y. 2010, PhRvD, 82, 043002

Ahlers, M. 2011, PhRvD, 84, 063006

Aleksić, J., Antonelli, L., Antoranz, P., et al. 2010, A\&A, 524, A77

Ambrogi, L., De Oña Wilhelmi, E., \& Aharonian, F. 2016, APh, 80, 22

Archambault, S., Archer, A., \& Benbow, W. 2017, ApJ, 835, 288

Arlen, T. C., Vassilev, V. V., Weisgarber, T., Wakely, S. P., \& Yusef Shafi, S. 2014, ApJ, 796, 18

Bertone, S., Vogt, C., \& Enßlin, T. 2006, MNRAS, 370, 319

Blasi, P., Burles, S., \& Olinto, A. V. 1999, ApJL, 514, L79

Broderick, A. E., Chang, P., \& Pfrommer, C. 2012, ApJ, 752, 22

Caprini, C., \& Gabici, S. 2015, PhRvD, 91, 123514
Carosi, A., Lucarelli, F., Antonelli, L. A., \& Giommi, P. 2016, Proc. SPIE, 9913, 99131Y

Chen, W., Buckley, J. H., \& Ferrer, F. 2015, PhRvL, 115, 211103

CTA 2017, Performance Files, https://portal.cta-observatory.org/CTA_ Observatory/performance/SitePages/Home.aspx

Domínguez, A., Primack, J. R., Rosario, D. J., et al. 2011, MNRAS, 410, 2556

Durrer, R., \& Neronov, A. 2013, A\&ARv, 21, 62

Eungwanichayapant, A., \& Aharonian, F. 2009, IJMPD, 18, 911

Fallon, L. 2010, in Proc. 25th Texas Symp. Relativistic Astrophysics (Heidelberg: SISSA), Session P6, doi:10.22323/1.123.0192

Fernandez Alonso, M. 2014, BAAA, 56, 347

Fernandez Alonso, M., Supanitsky, A. D., \& Rovero, A. C. 2015, Proc. ICRC, 34, 791

Finke, J. D., Reyes, L. C., Georganopoulos, M., et al. 2015, ApJ, 814, 20

Finnegan, G. 2011, arXiv:1111.0121

Gilmore, R. C., Somerville, R. S., Primack, J. R., \& Domínguez, A. 2012, MNRAS, 422, 3189

Gould, R., \& Rephaeli, Y. 1978, ApJ, 225, 318

Gould, R., \& Schréder, G. 1966, PhRvL, 16, 252

H.E.S.S. Collaboration, Abramowski, A., Acero, F., et al. 2010, A\&A, 520, A83

Kachelrieß, M., Ostapchenko, S., \& Tomàs, R. 2012, CoPhC, 183, 1036

Kotelnikov, E., Rubtsov, G., \& Troitsky, S. 2015, MNRAS, 450, L44

Meyer, M., Conrad, J., \& Dickinson, H. 2016, ApJ, 827, 147

Neronov, A., \& Semikoz, D. V. 2009, PhRvD, 80, 123012

Pshirkov, M. S., Tinyakov, P. G., \& Urban, F. R. 2015, MNRAS, 452, 2851

Takahashi, K., Inoue, S., Ichiki, K., \& Nakamura, T. 2011, MNRAS, 410, 2741

Vincent, P. \& H.E.S.S. Collaboration 2004, in Proc. SF2A-2004, Semaine de l'Astrophysique Francaise, ed. F. Combes et al. (Paris: EDP Science), 407

Wakely, S. P., \& Horan, D. 2008, Proc. ICRC, 3, 1341 